\title{
Reporting an Experience on the Establishment of a Quality Model for Systems-of-Systems
}

\author{
Daniel Soares Santos*, Brauner R. N. Oliveira*, Adolfo Duran ${ }^{\dagger}$, and Elisa Yumi Nakagawa* \\ *ICMC, Department of Computer Systems \\ University of São Paulo, São Carlos, SP, Brazil. \\ ${ }^{\dagger}$ DCC, Department of Computer Science \\ Fraunhofer Project Center \\ Federal University of Bahia, Salvador, BA, Brazil. \\ \{danielss, brauner\}@usp.br, adolfo@ufba.br, elisa@icmc.usp.br
}

\begin{abstract}
Currently, Systems-of-Systems (SoS) have performed an important role in diverse application domains, with representative examples in airport, military, and smart-cities, including crisis/emergency management. SoS refer to complex software-intensive systems, resulted from interoperability of independent constituent systems, performing new missions that could not be performed by any constituents working separately. For these critical systems, their quality is undoubtedly essential. However, in general, there is a lack of studies that discuss how quality has been addressed in such systems. The main contribution of this paper is to present an experience of establishing a quality model (i.e., a set of quality characteristics/attributes, subcharacteristics, and metrics) for SoS, in particular, for the crisis/emergent management domain. This quality model is based on ISO/IEC 25010 and it has also proved to be an important support to evaluate a system of this domain, however their construction must be performed with caution. Experience such as presented in this work could be repeated in other domains, contributing to improve the quality of a diversity of critical, complex SoS that are currently being built.
\end{abstract}

\section{INTRODUCTION}

प]

Software-intensive systems have become increasingly large and complex and even essential to the whole society. These systems are sometimes resulting from interoperability of constituent systems that work together to provide more complex missions that could not be completed by any of these systems separately [1]. This new class of softwareintensive systems has been referred as System-of-Systems (SoS) and can be found in different application domains, including medicine, airport, robotics, avionics, healthcare, and automotive [2], [3]. Currently, the development of SoS still presents great challenges for the classical software engineering [4], as they presents a set of unique characteristics. Moreover, these systems must assure high level of quality considering their use in diverse critical application domains.

In another perspective, software quality has been a research topic widely researched over the last three decades
[5]. In this context, a well-accepted way to support quality control is to adopt software quality models. A quality model intends to make the software quality better understandable and manageable. A widely known model is the ISO/IEC 25010 quality model that has become an international standard for evaluating quality of modern software-intensive systems 6]. ISO/IEC 25010 is based on the fact that the software product quality can be specified and evaluated using a hierarchical structure of quality attributes/characteristics (e.g., reliability and performance), sub-characteristics (e.g., availability and adaptability), and metrics to measure these characteristics and sub-characteristics [7]. Specifically for the context of SoS, in spite of the great necessity of dealing with software quality during their development and evolution, there are not quality models for SoS that can contribute to control and improve the quality of these systems. Additionally, due to the generic nature of the ISO/IEC standard, hierarchical quality models can be tailored upon its characteristics [8]

Motivated by this scenario, the main contribution of this paper is to present an experience in establishing a quality model for SoS in the crisis/emergency management domain from the ISO/IEC 25010. This quality model has been built in the context of a large international research project the RESCUER project 1 — which has as a main goal to develop an SoS for that domain, which intends to bring a clear impact and direct, innovative benefits to the society. Additionally, we conducted an evaluation of this SoS using this quality model. Results achieved until now show the valorous role of a quality model to improve quality of SoS. However, the building a quality model for this domain must be performed with attention mainly when it is strictly based in a standard such as the ISO/IEC 25010.

The remainder of this paper is organized as follows. Section II presents the background on SoS and quality models. Section III presents the establishment of the quality model. Section IV presents the application of this model. Section V discusses on learned lessons. Finally, Section VI presents our conclusions and future work.

\footnotetext{
1 http://www.rescuer-project.org/
} 


\section{BACKGROUND}

This section presents important concepts related to SoS and quality models, aiming a better understanding about the topics covered in this paper.

Regarding to SoS, definitions and characterization of SoS have been increasing discussed and widespread in recent years. Despite the number of different definitions existing in the literature, there is still no universally recognized definition for SoS, and then, their characterization depends often on the viewpoint and system's context. In general terms, an SoS can be seen as a "supersystem" composed by complex and operationally independent systems working together to achieve higher goal [9]. According to Maier [1], an SoS can be identified and differentiated from monolithic systems by the presence of features such as: (i) Operational Independence: constituent systems are operationally independent and have their own goals, even when disconnected from the SoS; (ii) Managerial Independence: it means that each constituent system is developed and maintained by different organizations, with their own stakeholders, development teams, processes and resources; (iii) Evolutionary Development: each constituent system evolves independently and, therefore, the SoS must also evolve, where structures, functions, and purposes are added, removed, and modified; (iv) Emergent Behavior: it means that a new behavior that can not be provided by any constituent system working separately emerges; and (v) Geographical Distribution: constituent systems may be located in different places changing information among them.

SoS started to gain popularity mainly on military systems as a strategy to reach goals or deliver unique capabilities wherein a collaborative work of complex systems is needed 1, 4. Furthermore, SoS is migrating from traditional military domains to civil domains, such as smart homes, healthcare, crisis/emergency management, and several others. In particular, an SoS in the context of crisis/emergency management allows more efficient response to crisis and incidents through integration of police, firefighters, military, and medical systems.

Achieving quality in these systems is a quite difficult task, mainly because constituent systems are sometimes developed and maintained by different organizations, with their own stakeholders, development teams, processes, and resources [10], [11]. In this context, quality models could be used to identify relevant quality characteristics that can be further used to guide the development, evolution, and evaluation of these systems 6 .

A software quality model may support a better understanding about what quality is in the context of software systems, supporting diverse activities throughout system development cycle. This is done through the identification of quality characteristics that are exhibited by software systems and can be aggregated to compose the overall software quality concept. These characteristics are generally called quality attributes (e.g., maintainability, performance, and security), which are presented by quality models to define, assess and/or predict software quality 5. The first models emerged in the early days of the software engineering area and since then, quality models are still subject of research. The first standardized quality model was proposed in the international standard ISO/IEC 91261 [12] in 2001, which was revised and replaced by the ISO/IEC 25010 [7] in 2011.

The ISO/IEC 25010 provides two quality models: (i) a quality in use model that provides five quality characteristics concerning software under operation by its stakeholders and (ii) a product quality model that is composed of eight quality characteristics concerning the software system apart of its stakeholders. Both are supposed to be applied to any kind of computer system that includes a software product. Characteristics of both models are decomposed into sub-characteristics that can be measured. When every sub-characteristic is measured, it is considered that the characteristic is also measured by aggregation. Having every characteristic measured, the overall quality of the product is determined. In order to achieve this goal, one or more metrics are defined and applied to each sub-characteristic, resulting in a value that represents the degree to which it is present in the final product. The ISO 9126-2 [13] is an example of standard that presents metrics for measuring sub-characteristics, and may be used together with ISO 25010 to evaluate quality of a software product.

\section{Establishing the Quality Model}

Before we present the development of the quality model, we present the context where this model is being established and used. In the context of crisis/emergency management, the main challenge for an Emergency Command and Control Centre is to quickly obtain contextual information to answer an emergency and ensure the correct decisions. An appropriate response is essential to attenuate the occurrence of physical injuries as well as the negative outcome to the public image of the involved organizations. Decisions based on incorrect or late information have a great potential for causing more damage.

In parallel, the everyday use of mobile devices, such as tablets and smartphones, provides an enabling technology for building new software solutions for also the emergency domain. Exploring such devices as a communication tool, the RESCUER research project proposes the development of an interoperable computer-based solution to provide Command and Control Centres with real-time contextual information related to the emergency situation in industrial areas and in large-scale events. This solution relies on the collection, combination, and aggregation of crowdsourcing information.

The RESCUER solution comprises four main constituents systems:

- Mobile Crowdsourcing Solution (MCS) implements suitable context-sensitive mechanisms for eyewitnesses 
and operational forces carrying mobile devices to provide the Command and Control Centre with information about emergency situations, taken into consideration the behavior of people under stress situations. The users provide reports of the incidents with text, photos, and videos. Besides, the RESCUER application is able to send relevant information from device sensors without user interaction.

- Data Analysis Solution (DAS) is composed by algorithms that process and filter the received data (e.g. image, text, and video) to extract relevant and consolidated information. This system is responsible for fusing similar data coming from different eyewitnesses in order to extract information such as the type of incident, the position and dimensions of the affected area, people density, evacuation routes, and possible approach routes for the first responders;

- Emergency Response Toolkit (ERTK) provides the Command and Control Centre with updated and relevant information, in an adequate format, to support decision-making during an emergency. It applies a set of solutions to manage the analyzed data coming from the DAS and presents them in a Real Time Dashboard, using adequate visualization means; and

- Communication Infrastructure (COM): supports the information flow between stakeholders even when traditional communication infrastructure is overloaded, by establishing Ad Hoc network communication to propagate data between users' phones and the command centre.

In the RESCUER platform, these constituents systems are part of an Integrated Solution (IS) that will gather crowdsourcing information and provide relevant information to the command and control centre. Since the development and evaluation of each main constituent of the RESCUER platform is not sufficient to guarantee the quality of the whole system, the IS was considered like an independent system during the development and application of the quality model.

This project defines an iterative project lifecycle, in which each subsequent iteration builds on and improves the results of the previous one. The overall strategy divides the lifecycle into three iterations steps. Basically, the iterations have been defined according to the integration of functionality (basic functions first, integration of more complex capabilities later). This facilitates the quality management, since it allows the quality evaluation of the first results and the gradual specification and maturation of the requirements of the RESCUER solution.

In this context, it can be noted that the solution emerged from the integration of described constituents systems, as well as the context development of the RESCUER solution is considered an SoS since can be perceived all the main SoS characteristics.

As early mentioned, to support RESCUER solution development, a quality model based on the ISO/IEC 25010
[7 has been established. This quality model determines quality characteristics and sub-characteristics to be considered during the three iterations of RESCUER project as well as a set of quality metrics to measure each quality characteristic. In the next sections, the establishment of the quality models is detailed concerning the quality attributes selection and quality metrics definition.

\section{A. Quality Attributes Selection}

To determine which quality characteristics and subcharacteristics are relevant to the RESCUER solution, all its non-functional requirements as well as the project goals and scope were carefully analyzed. This analysis allowed to translate each non-functional requirement into ISO/IEC 25010 quality characteristics, taking into account the Product Quality and Quality in Use models. To support this activity, it was performed a survey with requirement teams, developers, task leaders, and project coordinators in order to assure that all selected quality attributes are appropriate and relevant regarding the requirements of RESCUER solution. In this opportunity, we verified the applicability of the metrics and the viability of the application methods proposed by the ISO/IEC 9126 (it is detailed in the next section). Additionally, suggestions of other quality attributes that could be considered in the quality model and other metrics or application methods that could be used to measure the quality attributes were also gathered. After the questionnaires were answered, a meeting was performed with the stakeholders in order to discuss the results and, consequently, to obtain a consensus about the element that will compose the quality model.

The involvement of the stakeholders was very important, since the requirements about the RESCUER solution are still being detailed in the current phase of the project. Therefore, some quality attributes can still not be directly translated from the RESCUER requirements. Moreover, this strategy allows to obtain a consensus about all elements that compose the quality model, besides to assure that the main decisions about the quality model were coherent with the systems requirements and project goals.

On the order hand, it is important to highlight that not all quality characteristics and sub-characteristics are relevant for all constituents systems and as well as the IS. Depending on the use purpose of the quality model (system specification or evaluation), and the considered evaluation subject, a different subset of characteristics/subcharacteristics can be chosen accordingly to the specific goals and objectives. In addition, as RESCUER is an ongoing research project, its requirements will be probably modified during, and thus the quality model will be improved. Therefore, other quality characteristics that were not considered in the first project iteration, such as performance and security, will be added in the following project iterations. Figure 1 presents the quality characteristics and sub-characteristics selected to compose the developed quality model. 


\begin{tabular}{|c|c|c|c|c|c|c|}
\hline $\begin{array}{l}\text { Quality } \\
\text { Character. }\end{array}$ & $\begin{array}{l}\text { Quality Sub } \\
\text { Character. }\end{array}$ & Metric & Purpose of the metric & Method of application & $\begin{array}{l}\text { Interpretation of } \\
\text { measured value }\end{array}$ & $\begin{array}{l}\text { Artifact or } \\
\text { Data Source }\end{array}$ \\
\hline \multirow[t]{2}{*}{ Usability } & \multirow[t]{2}{*}{ Learnability } & USM3 & $\begin{array}{l}\text { What proportion of users } \\
\text { can operate successfully } \\
\text { a function without a } \\
\text { demonstration or tutorial? }\end{array}$ & $\begin{array}{l}\text { Number of users } \\
\text { that adequately operated } \\
\text { the functions by total } \\
\text { number of users }\end{array}$ & $\begin{array}{l}\text { The closer to } 1.0 \text {, } \\
\text { the better }\end{array}$ & $\begin{array}{l}\text { User test, interview } \\
\text { or user behavior } \\
\text { observation }\end{array}$ \\
\hline & & USM4 & $\begin{array}{l}\text { What proportion of } \\
\text { user can operate } \\
\text { successfully a function after } \\
\text { a demonstration or tutorial? }\end{array}$ & $\begin{array}{l}\text { Number of users that } \\
\text { adequately operated } \\
\text { the functions by total } \\
\text { number of users }\end{array}$ & $\begin{array}{l}\text { The closer to } 1.0 \text {, } \\
\text { the better }\end{array}$ & $\begin{array}{l}\text { User test, interview } \\
\text { or user behavior } \\
\text { observation }\end{array}$ \\
\hline
\end{tabular}

Table I: Examples of Metrics
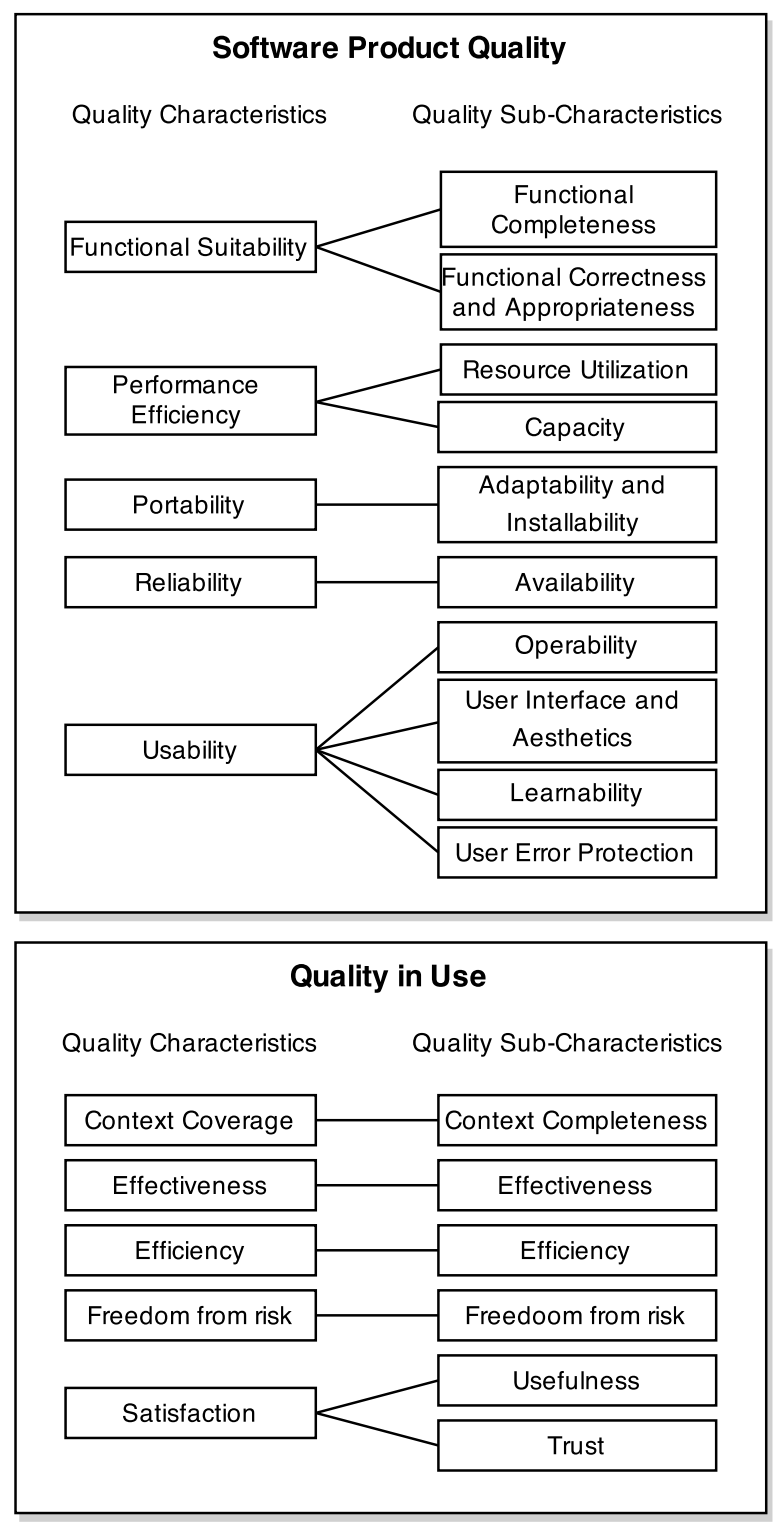

Figure 1: Established Quality Model

\section{B. Metrics Definition}

As early mentioned, quality metrics are used to measure the quality of a software product by measuring its quality attributes. When applying a quality metric, it is possible to obtain a quantitative value that characterizes the degree of compliance of the software to the corresponding quality characteristic. In this sense, for each quality subcharacteristics defined in the quality model, a set of appropriated metrics for their measurement was established. These metrics were selected and adapted from the international standards ISO/IEC 9126-2 - External Metrics [13] and ISO/IEC 9126-4 - Quality In Use Metrics [14. External metrics are used to measure the quality of the software product by measuring the behavior of the system, during testing stages or system operation. On the order hand, quality in use metrics are applied in a realistic system environment to verify if a product meets the needs of specified users to achieve their goals [14].

Table I presents examples of two metrics, USM3 and USM 4 - USability Metric 3 and 4, of a total of 20 metrics that we have established in the quality model. These were used to measure the learnability of MCS, a key quality characteristic since no training material should be necessary for the user to understand and interact with the RESCUER system during an emergency incident, even when users are under high stress situations. In this sense, these metrics are important to identify the influence of the demonstration or tutorial in the effectiveness of the users and consequently measure the level of learnability of the RESCUER solution. In addition, it is very important to highlight that specific input data is needed for an adequate application of these metrics. Input data can be obtained by using questionnaires, checklists, experiments, observations, etc. For each established metric, the method of application and the source of data or artifacts that could be used in the measurement were established. These source of data and artifacts, as well as the strategies that will be used to obtain the needed information to application of metrics, were properly detailed in evaluation plans created to guide the constituent systems and IS evaluation. Next section will present more details about the evaluation and the application of the quality model.

\section{Application of the Quality Model}

The developed quality model was used as basis to the evaluation of ERTK and MCS constituent systems. Each system was evaluated in four different situations, in Brazil and in Germany, taking into account the contexts of large events (FIFA World Cup 2014 and football games in Germany) and industrial areas (Camaçari Industrial 


\begin{tabular}{|c|c|c|c|c|c|c|c|c|}
\hline \multirow{2}{*}{$\begin{array}{l}\text { Quality } \\
\text { Character. }\end{array}$} & \multirow{2}{*}{$\begin{array}{l}\text { Quality Sub } \\
\text { Character. }\end{array}$} & \multirow{2}{*}{ Metric } & \multicolumn{2}{|c|}{ First Iteration } & \multicolumn{2}{|c|}{ Second Iteration } & \multicolumn{2}{|c|}{ Third Iteration } \\
\hline & & & Sub System & Acceptance Criteria & Sub System & Acceptance Criteria & Sub System & Acceptance Criteria \\
\hline \multirow[t]{3}{*}{ Usability } & \multirow[t]{3}{*}{ Learnability } & USM3 & $\begin{array}{l}\text { Mobile } \\
\text { Crowdsourcing } \\
\text { Solution }\end{array}$ & $\begin{array}{l}60 \% \text { of the users } \\
\text { should adequately } \\
\text { use the app without } \\
\text { demonstration }\end{array}$ & $\begin{array}{l}\text { Mobile } \\
\text { Crowdsourcing } \\
\text { Solution }\end{array}$ & $\begin{array}{l}65 \% \text { of the users } \\
\text { should adequately } \\
\text { use the app without } \\
\text { demonstration }\end{array}$ & $\begin{array}{l}\text { Mobile } \\
\text { Crowdsourcing } \\
\text { Solution }\end{array}$ & $\begin{array}{l}70 \% \text { of the users } \\
\text { should adequately } \\
\text { use the app without } \\
\text { demonstration }\end{array}$ \\
\hline & & \multirow[t]{2}{*}{ USM4 } & $\begin{array}{l}\text { Mobile } \\
\text { Crowdsourcing } \\
\text { Solution }\end{array}$ & $\begin{array}{l}70 \% \text { of the users } \\
\text { should adequately } \\
\text { use the app with } \\
\text { demonstration }\end{array}$ & $\begin{array}{l}\text { Mobile } \\
\text { Crowdsourcing } \\
\text { Solution }\end{array}$ & $\begin{array}{l}75 \% \text { of the users } \\
\text { should adequately } \\
\text { use the app with } \\
\text { demonstration }\end{array}$ & $\begin{array}{l}\text { Mobile } \\
\text { Crowdsourcing } \\
\text { Solution }\end{array}$ & $\begin{array}{l}80 \% \text { of the users } \\
\text { should adequately } \\
\text { use the app with } \\
\text { demonstration }\end{array}$ \\
\hline & & & $\begin{array}{l}\text { Emergency } \\
\text { Response } \\
\text { Toolkit }\end{array}$ & $\mathrm{N} / \mathrm{A}$ & $\begin{array}{l}\text { Emergency } \\
\text { Response } \\
\text { Toolkit }\end{array}$ & $\begin{array}{l}50 \% \text { of the users } \\
\text { should adequately } \\
\text { use the app with } \\
\text { demonstration }\end{array}$ & $\begin{array}{l}\text { Emergency } \\
\text { Response } \\
\text { Toolkit }\end{array}$ & $\begin{array}{l}75 \% \text { of the users } \\
\text { should adequately } \\
\text { use the app with } \\
\text { demonstration }\end{array}$ \\
\hline
\end{tabular}

Table II: Evaluation Plan

Complex ${ }^{2}$ in Brazil). A total of 172 people participated of our evaluation. For this, a general evaluation plan was first developed in order to guide the evaluation of all constituents systems, including the IS, during the three iterations of the project. This general plan defines a set of assessment criteria that will be used to decide whether the metric results are satisfactory or not, considering the expected results for each project iteration. In general, these criteria are numerical thresholds or targets used to determine the need for action or further investigation. This allowed us to identify and, therefore, react in a straightforward manner to the problems that influenced the overall quality of the system. These criteria were defined through detailed analysis of the RESCUER quality requirements and refined by the requirements team, task leaders, and project coordinators.

To better manage and control the quality evolution of the RESCUER system, a different set of assessment criteria was established for each iteration. The assessment criteria were defined considering an increased level of rigor, since the metric results must improve in the course of the iterations in order to achieve the quality requirements expected to be in the final of the project. Table II presents the assessment criteria defined for the metrics USM3 and USM4, and the increasing of the rigor level of each assessment criteria during the three iterations.

For each constituent system, it was developed a specific evaluation plan to define and detail the set of strategies, source data and artifacts that will be used to obtain the needed input information for the application of the quality metrics, and, therefore, to obtain the final result about the compliance of the RESCUER constituent systems with the quality attributes established in the quality model. Since the evaluation focus of the first iteration was the usability and user experience, the strategy was basically to ask the participants to use the system following a set of key tasks while their behavior was observed in order to identify if each task was performed following an expected way. In addition, a brief user interview was performed to identify the system acceptance and aspects regarding the user experience. In the specific evaluation plan were detailed all the forms and questionnaires that were applied to evaluators and

\footnotetext{
2 http://www.coficpolo.com.br/
}

participants in the execution of the evaluation.

In general, the application of the quality model can be summarized as following: (i) in the first step, from the quality model, it was selected the quality attributes and metrics established for the MCS addressing the first project iteration; (ii) in the second step, each quality characteristic/sub-characteristic was measured through the application of the metrics; and (iii) finally, the results were compared with the assessment criteria to identify if the quality characteristics were achieved and, consequently, to act in the quality characteristics that have not been sufficiently achieved.

Table III presents the evaluation results regarding the quality attributes defined for MCS in the first iteration of the project. The results were satisfactory considering our expectations for the first evaluation iteration of the RESCUER project. This means that, taking into consideration the average in all evaluation places, results were higher than the values of the assessment criteria. The quality model facilitated the identification of factors that can impact specific quality attributes of the system, as well as the quality of RESCUER solution as a whole. Through this first evaluation iteration and the feedback provided, it was observed that the RESCUER solution can be refined to achieve a higher quality for the next evaluation iteration and that there is still room for improvement in order to make the solution as intuitive as possible. However, the establishment of a quality model in the SoS context imposes some challenges as those described in the next section.

\section{Learned Lessons and Discussions}

The establishment and use of a quality model in the SoS context impose several challenges and difficulties mainly due to the SoS characteristics, such as managerial independence, evolutionary development, and geographical distribution. In the RESCUER project, these characteristics have proven to significantly impact the productivity, success, and effort required for the establishment of a quality model. In addition, the current quality models such as the ISO/IEC 25010 have several limitations that difficult its application in the SoS context, mainly because of the lack of clearly decomposition criteria that determine how the quality attributes achieved in the constituent systems can impact and determine the SoS quality as a whole [15]. 


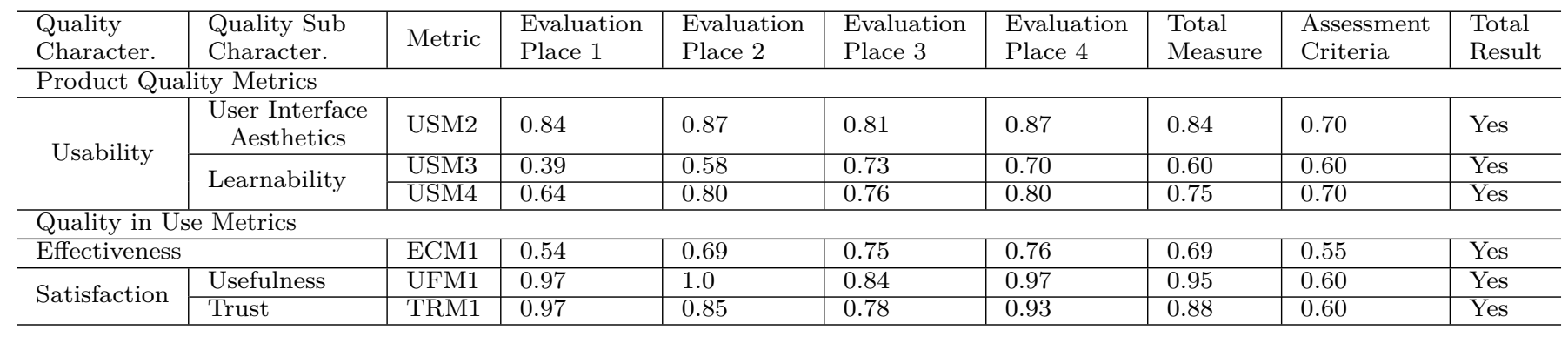

Table III: Evaluation Results of the Mobile Crowdsourcing Solution

This is a very complex problem, since quality attributes not achieved in one of their constituents can impact on the quality of other constituent systems. In addition, this impact depends on the role and importance that each constituent system plays in an SoS.

In addition, it was observed that the establishment of domain-specific quality models based on general quality models must be performed with attention. There are, for instance, domain-specific quality attributes that are not present in ISO/IEC 25010. This could be mitigated by using methodologies such as the one presented in [8], which considers external domain sub-characteristics to the general quality model.

In a parallel study, we found that the coverage rate of the ISO/IEC 25010 is only $44 \%$ regarding the quality attributes important for SoS [15]. This may significantly compromise the completeness and comprehensiveness of the quality model that have been developed. Moreover, some well-established definitions for each quality attribute can not be fully applied in the SoS context due to the flexible, dynamic nature of these systems. Therefore, some quality attributes defined in the ISO/IEC 25010 such as reliability can not directly express the required characteristics for RESCUER project and possibly others SoS.

On the other hand, it is important to say that during the refinement of the presented quality model in the next two iterations, other important key SoS quality attributes such as interoperability, security, reliability, and performance, including those ones identified in [15, will be considered.

In this sense, we expect that this quality model, with the expected improvements, can adequately guide the development and evaluation of the RESCUER solution, and our experience establishing it light the construction of quality models for domains where SoS have been applied.

\section{Conclusions}

SoS is becoming increasingly important and being applied in several critical sectors of the society. By their criticality, evaluation of their quality is essential. In this scenario, this paper presented an experience of establishing a quality model for SoS, in particular, for the domain of crisis/emergency management. In addition, we applied this model in a case study to evaluate an SoS of such domain. As a result, we observed that quality models must be adopted as one of the main guidelines to support the improvement of quality of software-intensive systems, including SoS. For the future work, we intend to apply this quality model in other evaluation iterations, as well as to update it to be consolidate as a model to be adopted for this critical, essential application domain. Besides that, we intend our experience can be reproduced in other critical domains where SoS are found.

\section{ACKNOWLEDGMENTS}

This work is supported by Brazilian funding agencies FAPESP (Grant: 2014/02244-7), CNPq (Grant: 490084/2013-3).

\section{REFERENCES}

[1] Mark W. Maier. Architecting principles for systems-of-systems. Systems Engineering, 1(4):267 - 284, 1998.

[2] Elisa Y. Nakagawa, Marcelo Gonçalves, Milena Guessi, Lucas B. R. Oliveira, and Flavio Oquendo. The State of the Art and Future Perspectives in Systems of Systems Software Architectures. In SESoS, pages 13-20, Montpellier, France, 2013.

[3] J. A. Lane. What is a system of systems and why should i care? Technical report, USC-CSSE, 2013.

[4] Department of Defense. Dodaf architecture framework version 2.02. http://cio-nii.defense.gov/sites/dodaf20/, 2010. (Accessed 20/03/2015).

[5] S. Wagner. Software Product Quality Control. Springer, Berlin, Heidelberg, 2013.

[6] N. Azizian, T. Mazzuchi, S. Sarkani, and D. F. Rico. A Framework for Evaluating Technology Readiness, System Quality, and Program Performance of U.S. DoD Acquisitions. Syst. Eng., 14(4):410-426, 2011.

[7] ISO/IEC. ISO/IEC 25010 - Systems and software engineering - Systems and software Quality Requirements and Evaluation (SQuaRE) - System and software quality models. Technical report, 2011.

[8] Xavier F. and Carvallo, J. P. Using Quality Models in software package selection. IEEE Software, 20(1):34-41, 2003.

[9] M. Jamshidi. Systems of Systems Engineering: Principles and Applications. Taylor \& Francis, 2008.

[10] M. Gagliardi, W. Wood, J. Klein, and J. Morley. A uniform approach for system of systems architecture evaluation. Cross Talk, $22(3-4): 12-15,2009$.

[11] D. S. Santos, B. Oliveira, M. Guessi, F. Oquendo, M. Delamaro, and E. Y. Nakagawa. Towards the evaluation od system of systems softaware architecture. In WDES, pages 1-6, Maceio, Brasil, 2014.

[12] ISO/IEC. ISO/IEC 9126. Software engineering - Product quality, 2001.

[13] ISO/IEC. ISO/IEC 9126 - Software engineering - Product quality - Part 2: External metrics, 2003.

[14] ISO/IEC. ISO/IEC 9126 - Software engineering - Product quality - Part 4: Quality in Use metrics, 2003.

[15] D. S. Santos, T. Bianchi, K. R. Felizardo, and E. Y. Nakagawa. An investigation on quality attributes of systems-of-systems. Technical report, São Paulo, Brazil, 2015. 